VOL. XV, PAGES 707-728 (1973)

\title{
Experimental and Mathematical Modeling Studies of Protozoan Predation on Bacteria
}

\author{
R. P. CANALE, Department of Civil Engineering, The University of \\ Michigan, Ann Arbor, Michigan, T. D. LUSTIG, School of Law, \\ University of Colorado, Boulder, Colorado, P. M. KEHRBERGER, \\ Hydroscience, Inc., Westwood, New Jersey, and J. E. SALO, The \\ State of Massachusetts, Division of Water Pollution Control, Arlington, \\ Massachusetts
}

\section{Summary}

The predation of bacteria by protozoan in both continuous and batch cultures was studied using experimental and modeling techniques. The predator organism was the ciliate, Tetrahymena pyriformis. The bacterium, Aerobacter aerogenes, served as the prey. Several batch growth responses were observed each initiated at a different nutrient level. Continuous cultures were conducted over a range of dilution rates. The models studied were partially successful in simulating the empirical data. Deviations between the model and the actual population behavior are discussed and possible explanations for the differences proposed.

\section{INTRODUCTION}

Engineers have long recognized the importance of predators in microbial systems. The activity of predators reduces the production of prey organisms which can result in incomplete substrate ultilization and suboptimal product formation. Under certain circumstances it is even possible that predators can completely destroy the prey. Although the actual extinction of the prey is rare in engineering cultures, predators may be a significant factor influencing reactor performance. For example, it is well known that heavy rotifer predation in oxidation ponds can decrease the rate of stabilization of waste influents. Further, predators may reduce the stability of continuous cultures following pertabations in temperature, dilution rate, or feed composition.

Bacterial predators are an important component of the microbial community operative in common biological waste treatment operations. Their major beneficial role involves the clearing of dispersed

(C) 1973 by John Wiley \& Sons, Inc. 
bacterial cells which results in increased treatment effectiveness. As members of complex food webs, algal and bacterial predators act to transfer energy to organisms on higher trophic levels. These roles of microbial predators are of direct interest to engineers seeking to curb eutrophication in lakes and reserviors and optimize biological production of selected members of food chains.

Two distinct types of problems arise with respect to the influence of predators in engineering cultures. In one case a defined controlled culture is maintained to perform a specific task, such as acting as part of a life support system for man during extended space flights. The invasion of predators into such cultures could have disastrous effects on man and thus it is important to be able to predict the effect of such invaders under various culture conditions. In other cases, uncontrolled biological cultures bring about chemical changes in the growth supporting medium. Here the role of predators as members of a diverse community must be identified. For example, the interpretation of laboratory Biochemical Oxygen Demand (BOD) data is closely associated with the secondary oxygen demand of protozoa. Also, the occurance of certain ciliated protozoan such as Vorticella in activated sludge has for many years been used to reflect the general state of health of the floc community.

This "indicator organism" technique has been a useful approach for predicting the likely behavior of mixed cultures in many applications. A second technique, which has been given much attention recently, involves the specification of mathematical models to represent the prey-predator interactions. The mathematical models usually take the form of a system of ordinary differential equations, the integration of which simulates the dynamic behavior of the population components. The simplest prey-predator models were first proposed by Lotka ${ }^{1}$ and Volterra. ${ }^{2}$

$$
\begin{aligned}
& \frac{d H}{d t}=\alpha H-\beta H P \\
& \frac{d P}{d t}=\gamma H P-\delta P
\end{aligned}
$$

where $H$ is the prey population density; $P$ is the predator population density; and $\alpha, \beta, \gamma, \delta$ are constants.

Equations (1) and (2) are elementary statements of the causal relationships which express qualitatively the interactions operative in a closed prey-predator system. The solution of eqs. (1) and (2) gives a nonsinusoidal oscillation between the prey and predator. 
This type of behavior in prey-predator communities has been explored by ecologists in a number of experimental and natural systems.

The Lotka-Volterra model suggests exponential growth of the prey in the absence of the predator and unrestricted growth of the predator at high prey densities. Several workers such as Drake et al., ${ }^{3}$ Canale, ${ }^{4}$ Bungay and Cline, ${ }^{5}$ and Yang and Weston ${ }^{6}$ have proposed modifications which allow saturation of both the prey and predator growth rate. These modifications are represented below as eqs. (3), (4), and (5). Equation (3) defines the continuity of the limiting nutrient in the growth medium in an open homogeneous system with prey, but not predator, utilization of nutrient. Equation (4) expresses the continuity of a prey organism such as a bacterium being limited by the supply of a nutrient as well as by the activity of a predator in an open homogeneous reactor being fed a sterile growth medium. Equation (5) represents the continuity of the predator growing on the prey population.

$$
\begin{gathered}
\frac{d S}{d t}=\frac{Q}{V}\left(S_{i}-S\right)-\frac{\hat{\mu}}{Y} \frac{S}{K_{S}+S} H \\
\frac{d H}{d t}=\hat{\mu} \frac{S}{K_{S}+S} H-\frac{\hat{\eta}}{W} \frac{H}{K_{N}+H} P-\frac{Q}{V} H \\
\frac{d P}{d t}=\hat{\eta} \frac{H}{K_{H}+H} P-\frac{Q}{V} \mathrm{P}
\end{gathered}
$$

In the above equations $Q$ is the volumetric flow of fluid into and out of the reactor; $V$ is the reactor volume; $S$ is the concentration of prey growth-limiting nutrient in the reactor; $S_{i}$ is the concentration of prey growth-limiting nutrient entering the reactor; $\hat{\mu}$ is the maximum specific growth rate of the prey; $Y$ is the yield of prey during depletion of $S ; K_{S}$ is the growth rate saturation constant for the prey; $\hat{\eta}$ is the maximum specific growth rate of the predator; $W$ is the yield of the predator during consumption of prey; and $K_{H}$ is the growth rate saturation constant for the predator.

The growth of the prey and prey nutrient uptake have been assumed to obey the formulations proposed by Herbert et al. ${ }^{7}$ The predator growth and prey uptake is simulated assuming a similar kinetic relationship. The experimental findings of Proper and Garver $^{8}$ with the bacterial predator Colpoda and Curds and Cockburn ${ }^{9}$ and Curds ${ }^{10}$ with Tetrahymena pyriformis support this type of formulation. 
Canale ${ }^{11}$ has shown that the transient solution of eqs. (3), (4), and (5) can approach steady states following damped oscillations or following exponential decays. With certain parameter values it is also known that steady oscillations about a singular point can occur which are independent of the initial conditions, i.e., limit cycle oscillations. Although these limit cycles resemble the oscillations predicted by the Lotka-Volterra model, Canale ${ }^{11}$ has discussed the superiority of eqs. (3), (4), and (5) based on their consistency with known biological mechanisms operative in prey-predator systems.

Drake et al. ${ }^{3}$ have experimentally verified the existance of certain of these solutions with the prey organism Aerobacter aerogenes using glucose as a limiting carbon source with the myxameba Dictyostelium discoideum serving as the bacterial predator. The purpose of the present paper is to make known additional experimental observations from both batch and continuous culture which can be used to further test the adequacy of models similar to eqs. (3), (4), and (5) to simulate the dynamics of simple bacterium protozoan cultures.

\section{EXPERIMENTAL METHODS}

The experiments were designed to allow observation of the dynamic interaction among prey nutrients, a prey, and a predator in as simple as possible biological system. Both continuous and batch cultures were examined. Undesirable complications were avoided by utilization of a culture which contained only one predator and one prey species. The bacterium Aerobacter aerogenes was selected for the prey. The free-swimming ciliated protozoan, Tetrahymena pyriformis Syngen 1, Strain D was selected for the predator.

The bacterial nutrients consisted of a $\mathrm{pH} 7.2$ buffered solution of inorganic salts and sucrose. Cerophyl extract was added to this solution to stimulate the protozoan feeding as suggested by Ducoff et al. ${ }^{12}$ Lustig ${ }^{13}$ has shown that this medium does not permit growth of the protozoan in the absence of bacterium. The temperature during all experiments was maintained at $25^{\circ} \mathrm{C}+0.2$. Continuous aeration of the cultures provided an adequate supply of oxygen for the micro-organisms. All equipment and solutions were autoclaved before use, and pure culture techniques were carefully applied throughout the experiments.

Samples withdrawn from both continuous and batch reactors were routinely analyzed for bacterial-limiting nutrient concentration, bacterial density, and protozoan biovolume. The bacterial-limiting nutrient in all cases was organic carbon, which was approximated as 
the carbohydrate remaining in solution after passing the samples through a 0.45 micron filter. The carbohydrate was determined using the anthrone reaction as described by Gaudy. ${ }^{14}$ Bacterial density was approximated by observations of the optical density of the culture suspension following differential centrifugation to remove the protozoan cells. Optical density (OD) measurements were made with a Bausch and Lomb Spectronic 20 model 4 spectrophotometer at $600 \mu$. The protozoan biovolume and cell size distribution were determined using a Coulter counter (Model B) which was calibrated using spheres of Iycopodium of known size. A detailed description of all equipment and methodology used in this paper can be found in a report by Lustig. ${ }^{13}$

\section{RESULTS}

\section{Batch Runs}

Nine batch runs were conducted. In most cases sterile growth medium was inoculated with fresh Aerobacter aerogenes. Following nutrient depletion and the maximum bacterial density, the culture was inoculated with fresh Tetrahymenia pyriformis. Censusing of the population continued four to five days until the start of the protozoan decline. The results of the batch runs are summarized in Table I. The maximum growth rates shown in Table I were calculated by measuring the maximum slope of the bacterium and protozoan concentration changes with time on semilog paper.

TABLE I

Summary of Batch Run Studies

\begin{tabular}{rccccc}
$\begin{array}{c}\text { Initial } \\
\text { Carbo- } \\
\text { hydrate, } \\
\text { mg/liter }\end{array}$ & $\begin{array}{c}\text { Minimum } \\
\text { Carbo- } \\
\text { hydrate }\end{array}$ & $\begin{array}{c}\text { Maximum } \\
\text { Bacterial } \\
\text { O.D. }\end{array}$ & $\begin{array}{c}\text { Maximum } \\
\text { Protozoan } \\
\text { Biovolume/ } \\
\text { ml }\end{array}$ & $\begin{array}{c}\text { Maximum } \\
\text { Bacterial } \\
\text { Specific } \\
\text { Growth } \\
\text { Rate, hr-1 }\end{array}$ & $\begin{array}{c}\text { Maximum } \\
\text { Protozoan } \\
\text { Specific } \\
\text { Growth } \\
\text { Rate, hr-1 }\end{array}$ \\
\hline 50 & 15 & 0.05 & 12,900 & 0.209 & 0.118 \\
64 & 12 & 0.046 & $\mathbf{1 7 , 5 0 0}$ & 0.309 & 0.111 \\
117 & 22 & 0.105 & $\mathbf{4 2 , 8 0 0}$ & 0.400 & 0.144 \\
122 & 12 & 0.170 & 42,900 & 0.368 & 0.160 \\
175 & 12 & 0.215 & $\mathbf{4 8 , 9 0 0}$ & 0.439 & 0.153 \\
228 & 14 & 0.281 & $\mathbf{6 1}, 500$ & 0.512 & $\mathbf{0 . 0 8 6}$ \\
265 & 20 & 0.265 & $\mathbf{7 7 , 9 0 0}$ & 0.512 & 0.124 \\
290 & $\mathbf{4 4}$ & 0.281 & $\mathbf{7 9 , 0 0 0}$ & 0.542 & $\mathbf{0 . 1 0 6}$ \\
306 & 26 & 0.367 & $\mathbf{9 0 , 7 0 0}$ & 0.542 & $\mathbf{0 . 1 1 9}$ \\
\hline
\end{tabular}


To insure that the Cerophyl extract, used to stimulate prey ingestion and reproduction of the protozoan, was present in nonlimiting concentrations, two batch runs were made at approximately equal total carbohydrate concentrations but differing Cerophyl concentrations. The run starting with an initial total carbohydrate of $290 \mathrm{mg} /$ liter contained $92 \mathrm{mg} /$ liter Cerophyl extract, while the run starting with an initial total carbohydrate concentration of 306 $\mathrm{mg} /$ liter contained only $30 \mathrm{mg} /$ liter of Cerophyl extract. The results in Table I do not suggest any accelerated growth of the Tetrahymena at Cerophyl concentrations above $30 \mathrm{mg} /$ liter. However, the residual carbohydrate following the bacterial growth did increase with an increased percentage of Cerophyl in the medium. Each batch run contained at least $30 \mathrm{mg} /$ liter Cerophyl, and thus the results are not biased by lack of protozoan growth factors.

Figure 1 shows a plot of the carbohydrate utilized during bacterial growth in each batch run versus the maximum optical density. The slope of the straight line which approximates the trend of these data results in a yield coefficient of $0.428 \mathrm{mg}$ of bacteria produced per milligram of total carbohydrate consumed. This number was calculated assuming one optical density unit is equivalent to 360

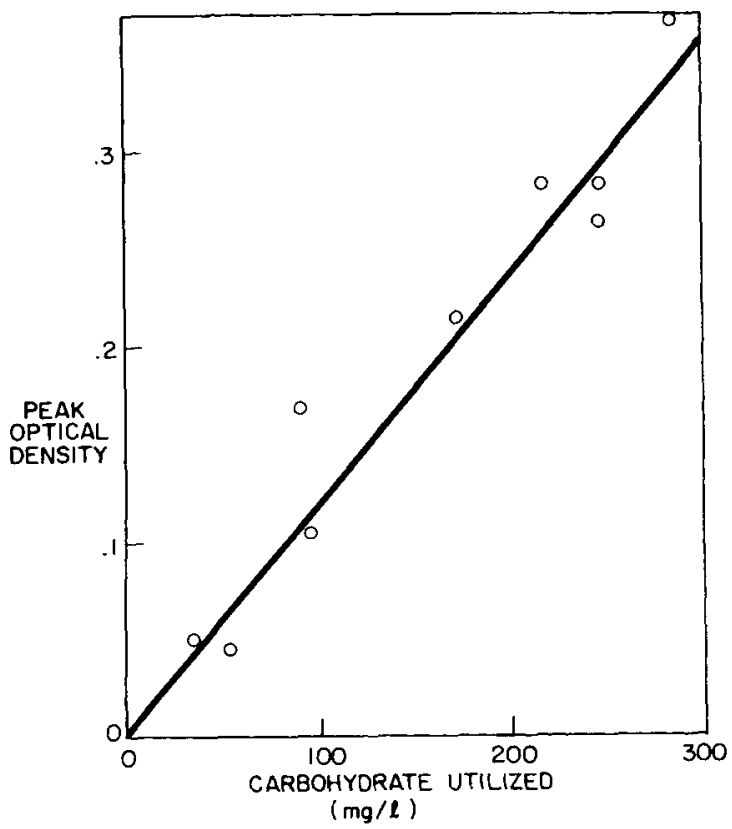

Fig. 1. Carbohydrate utilized during bacterial growth in batch culture. 
$\mathrm{mg} /$ liter of bacteria as reported by Lustig ${ }^{13}$. Figure 2 shows a plot of initial peak in optical density versus the maximum protozoan biovolume produced in each batch run. The slope of the line in Figure 2 is equivalent to a yield coefficient of $0.73 \mathrm{mg}$ of protozoan produced per milligram of bacterium consumed, assuming $10^{3}$ biovolume units are approximately equivalent to $10^{-3} \mathrm{mg}$ of protozoan as reported by Lustig. ${ }^{13}$

Figure 3 shows specific rates for Aerobacter aerogenes calculated by numerical differentiation of the batch run observations plotted against usable carbohydrate. Figure 4 shows similar results for the protozoan specific growth rate as a function of bacterium density. These plots or Lineweaver-Burk plots give an average maximum specific growth rate of $0.56 \mathrm{hr}^{-1}$, and a saturation constant of 16 $\mathrm{mg} /$ liter of carbohydrate for the bacterium growth, and an average maximum specific growth rate of $0.1 \mathrm{hr}^{-1}$ and a saturation constant of $6.1 \mathrm{mg}$ /liter of bacterium for the protozoan growth.

Figures 5 and 6 show a typical transient response in a batch culture following inoculation of bacterium, and subsequent to the bacterial maximum density, inoculation with protozoan. The total carbohydrate drops rapidly as a result of bacterium uptake to a minimum value of about $15 \mathrm{mg}$ /liter. Following this minimum, the value

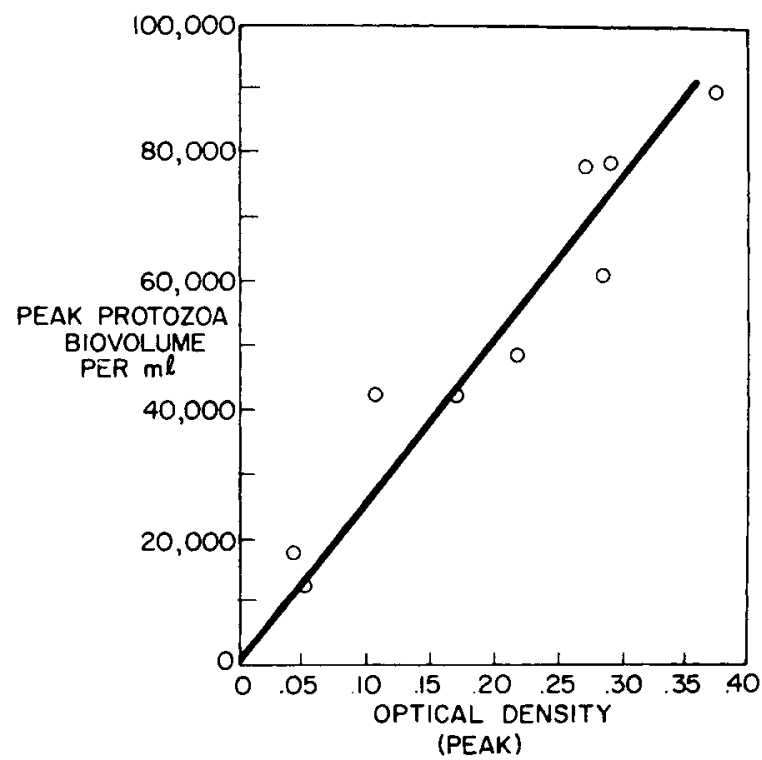

Fig. 2. Bacterium utilized during protozoan growth in batch culture. 


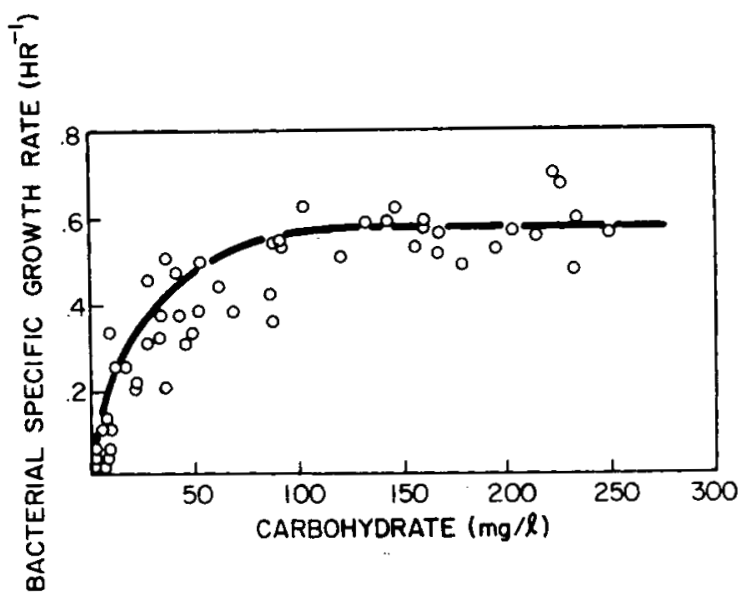

Fig. 3. Specific growth rate of Aerobacter aerogenes vs. soluble carbohydrate in medium.

slowly rises to about $40 \mathrm{mg} /$ liter near the end of the experiment. The bacterium increases during a growth phase and then declines as a result of the protozoan predation. The protozoan increase slowly at first to a peak value and then quickly enter a phase of declining biovolume. Figure 6 shows the increase in protozoan number parallel to, but lagging, the protozoan biovolume. The peak in cell number occurs $16 \mathrm{hr}$ after the biovolume peak. The relative

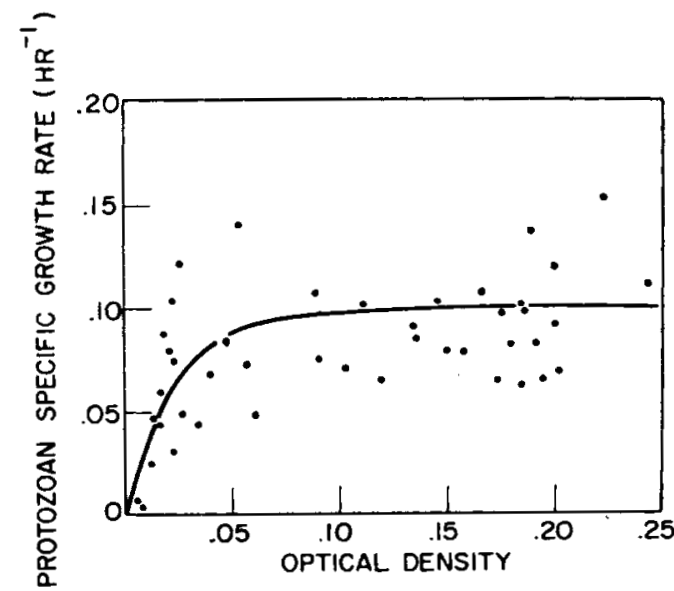

Fig. 4. Specific growth rate of Tetrahymena pyriformis vs. OD of bacterium in medium. 


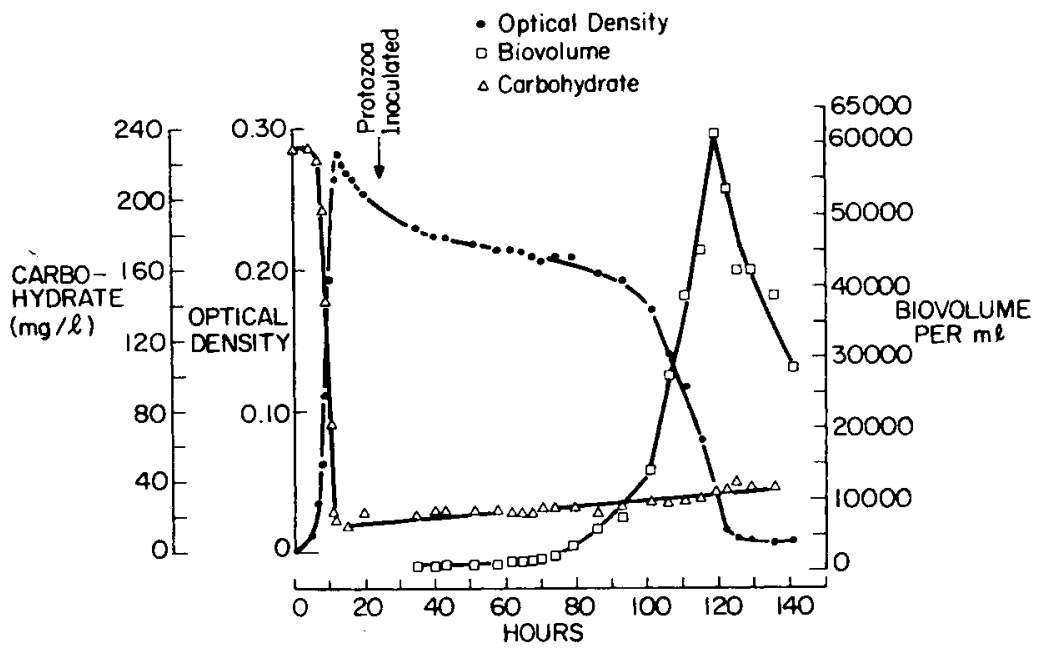

Fig. 5. Population and nutrient transients during batch culture.

cell size of the Tetrahymena at the peak biovolume is shown to be about four times the cell size at termination of the declining phase The rapid decrease in biovolume occurs at the expense of the cell size during a period when the rate of change of cell number is a maximum.

\section{Continuous Runs}

In all the continuous run studies sterile growth medium having a total carbohydrate concentration of $250 \mathrm{mg} /$ liter was supplied to the

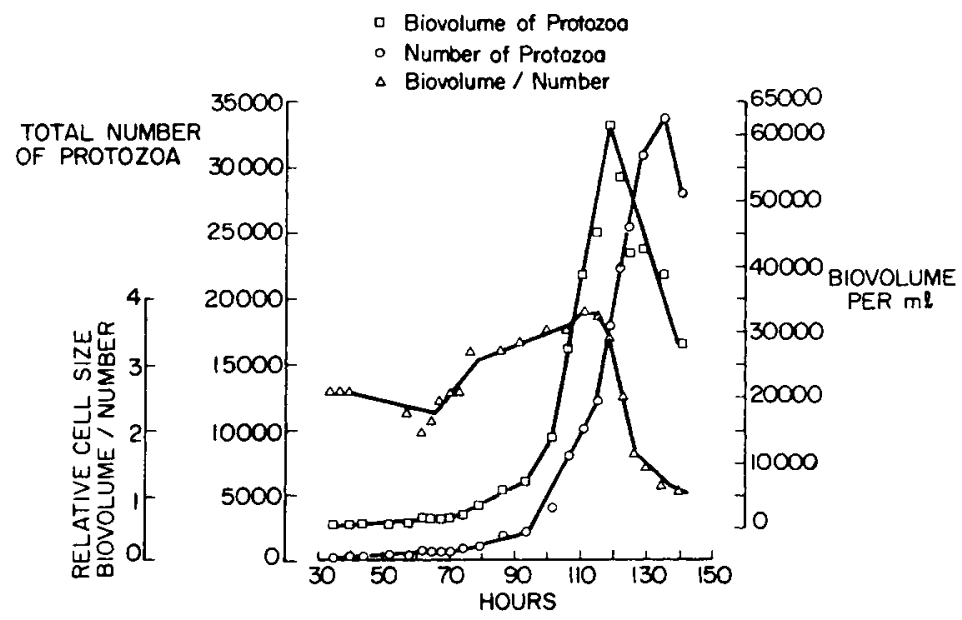

Fig. 6. Number and individual Tetrahymena size transients during batch culture. 
TABLE II

Summary of Continuous Run Studies

\begin{tabular}{cccl}
$\begin{array}{c}\text { Average Flow } \\
\text { Rate, } \mathrm{ml} / \mathrm{min}\end{array}$ & $\begin{array}{c}\text { Dilution Rate, } \\
\mathrm{hr}^{-1}\end{array}$ & $\begin{array}{c}\text { Duration of } \\
\text { Experiments, } \\
\mathrm{hr}\end{array}$ & Community Response \\
\hline 3.07 & 0.177 & 38 & washout of protozoa \\
2.36 & 0.136 & 103 & washout of protozoa \\
1.80 & 0.104 & 71 & uncertain protozoan washout \\
1.43 & 0.0825 & 393 & damped oscillation \\
1.24 & 0.0715 & 427 & damped oscillation \\
1.03 & 0.0595 & 312 & steady state \\
0.82 & 0.0473 & 442 & steady state followed by pro- \\
& & & tozoan lysis \\
\hline
\end{tabular}

reactor at seven different constant flow rates. A summary of the dilution rates and system responses is given in Table II. Figure 7 shows the soluble carbohydrate, optical density, and protozoan biovolume behavior at four dilution rates. At a dilution rate of 0.177 $\mathrm{hr}^{-1}$ the protozoan biovolume increased for a short time prior to an eventual decline. During the washout of the protozoan the optical density of the bacterium increased to a peak value of 0.225 , while the carbohydrate decreased from about $40 \mathrm{mg} /$ liter to about $8 \mathrm{mg} /$ liter.

- Optical Density

- Biovolume

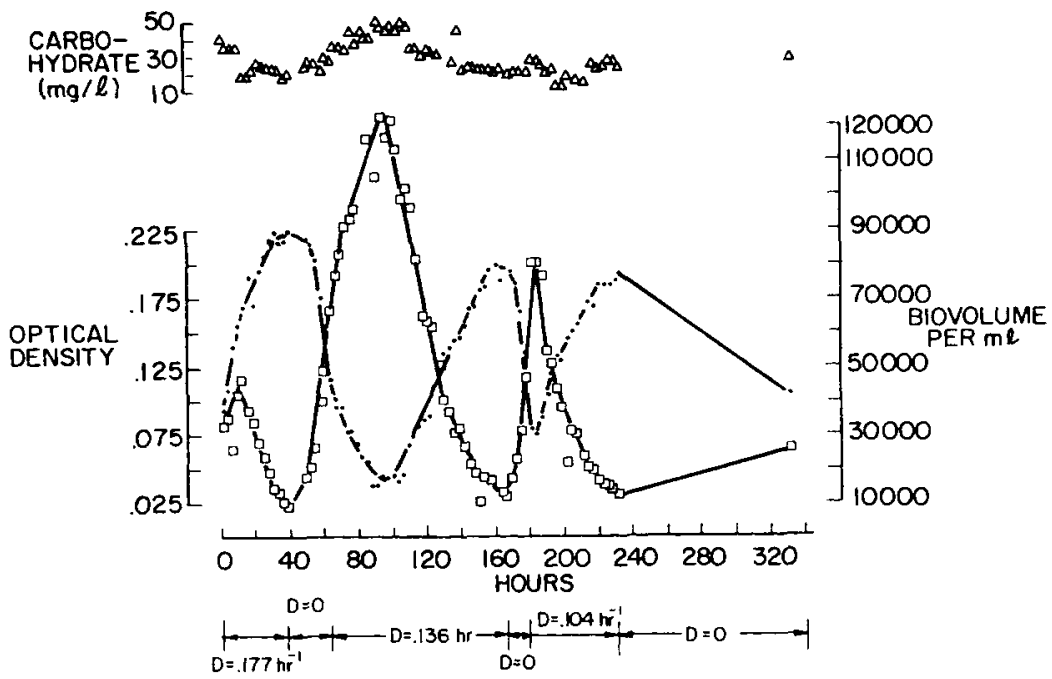

Fig. 7. Soluble carbohydrate, OD, and protozoan biovolume response during continuous culture at indicated dilution rate. 
When the dilution rate was reduced to zero the protozoan recovered rapidly increasing to a biovolume of approximately 65,000 per $\mathrm{ml}$. This increase in protozoan activity reduced the optical density to about 0.115 . Twenty-four hours after the dilution rate was reduced to zero, the pump was reactivated to deliver about $2.36 \mathrm{ml} / \mathrm{min}$ which was equivalent to a dilution rate of $0.136 \mathrm{hr}^{-1}$. The protozoan biovolume increased to about 120,000 per $\mathrm{ml}$ before entering a declining stage. The protozoan was observed for approximately $74 \mathrm{hr}$. The optical density varied inversely to the biovolume-decreasing to 0.03 at the protozoan peak and increasing to 0.2 at the termination of the protozoan decline. The carbohydrate varied directly with the protozoan biovolume and inversely to the optical density. At high optical densities the carbohydrate was less than $20 \mathrm{mg} /$ liter, while at low optical densities the carbohydrate reached a value of over 50 $\mathrm{mg}$ /liter. Following the protozoan decline during dilution at a rate of $0.136 \mathrm{hr}^{-1}$, the pump was again turned off to allow the protozoan to recover at the expense of the bacterium. One hundred and eighty hours after the start of the experiment, a dilution rate of $0.104 \mathrm{hr}^{-1}$ was established in the reactor. The protozoan again declined allowing the bacterium to peak at an optical density of 0.195 . The system behavior was observed for $71 \mathrm{hr}$.

Figure 8 shows the response of the community for $393 \mathrm{hr}$ during which the dilution rate was maintained at a constant value of 0.0825

- Optical Density

- Biovolume

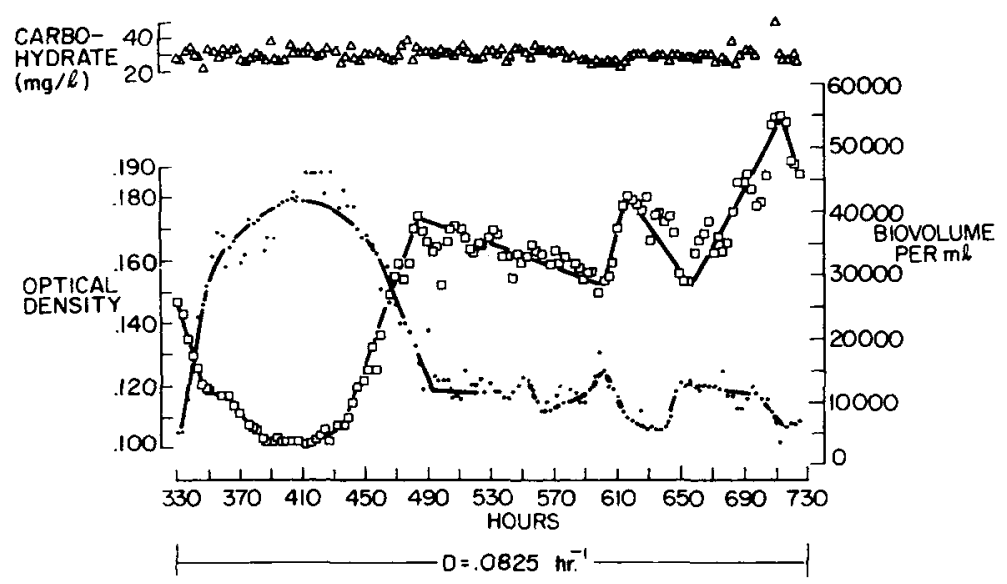

Fig. 8. Soluble carbohydrate, $O D$, and protozoan biovolume response during continuous culture at indicated dilution rate. 
$\mathrm{hr}^{-1}$. Following an initial decline, the protozoan biovolume gradually increased with irregular oscillations. The bacterium optical density varied inversely to the protozoan biovolume throughout the sampling period. The soluble carbohydrate values were generally low, with random perturbations about a mean value of about 25 $\mathrm{mg} /$ liter.

The variation of the carbohydrate, optical density, and biovolume for $427 \mathrm{hr}$ during a constant dilution rate of $.0715 \mathrm{hr}^{-1}$ is illuatrated in Figure 9. After an initial transient period the optical density appeared to approach a value of about 0.110 , while the carbohydrate fluctuated around $25 \mathrm{mg} /$ liter. The protozoan biovolume approached a value of about 30,000 per $\mathrm{ml}$. These results are similar to the results obtained at a dilution rate of $0.0825 \mathrm{hr}^{-1}$.

Figure 10 shows the changes of the system during a $312 \mathrm{hr}$ continuous run at a dilution rate of $0.0595 \mathrm{hr}^{-1}$. The data illustrate a steady state among the variables. The optical density maintained a value of about 0.09 which is somewhat lower than values obtained at higher dilution rates. The protozoan biovolume appeared to vary randomly between 30,000 and 35,000 per $\mathrm{ml}$. The soluble carbohydrate approached a value below those observed at higher dilution rates.
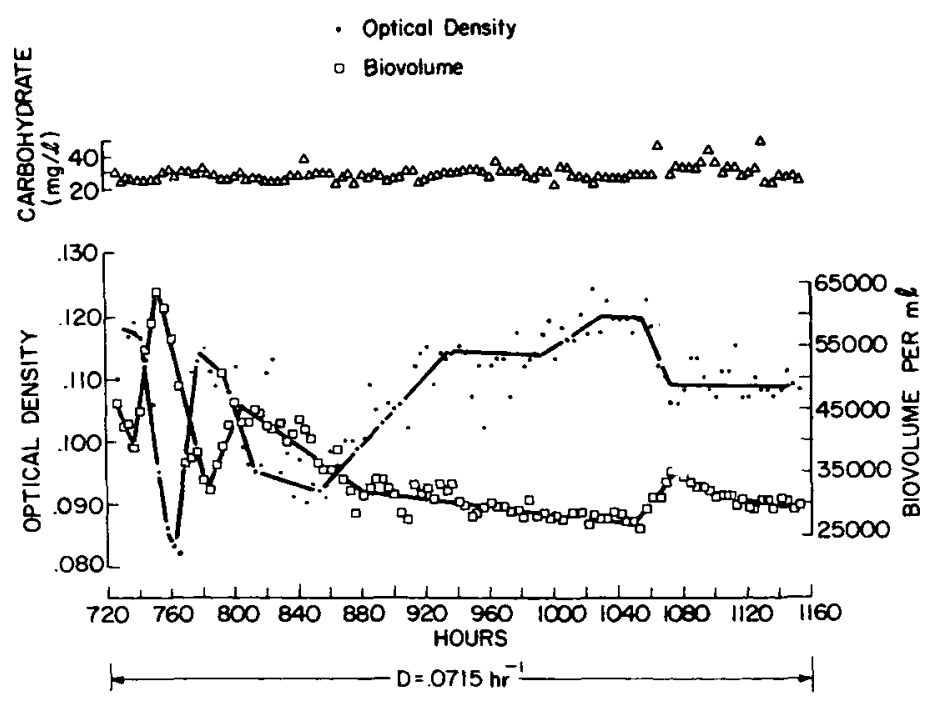

Fig. 9. Soluble carbohydrate, OD, and protozoan biovolume response during continuous culture at indicated dilution rate. 
- Optical Density

- Biovolume

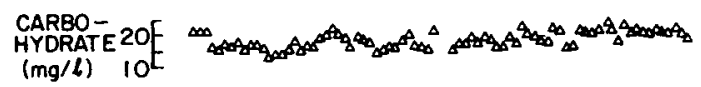

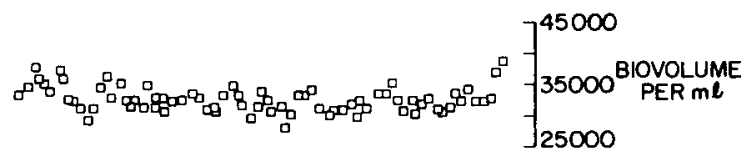

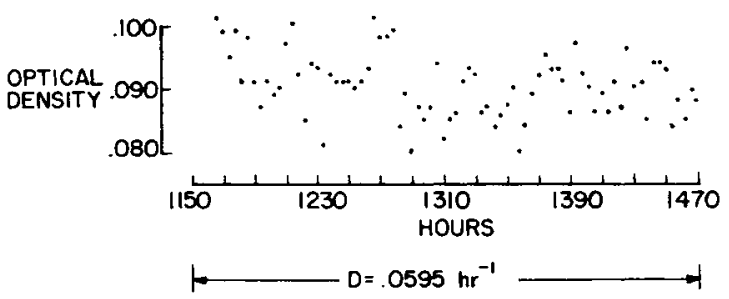

Fig. 10. Soluble carbohydrate, OD, and protozoan biovolume response during continuous culture at indicated dilution rate.

Figure 11 illustrates a steady state maintained for $170 \mathrm{hr}$ at a dilution rate of $0.0473 \mathrm{hr}^{-1}$. The optical density was nearly constant at a value of about 0.075 . The protozoan biovolume was between 35,000 and 40,000 per $\mathrm{ml}$. The carbohydrate appeared to increase slowly from $17 \mathrm{mg} /$ liter to $30 \mathrm{mg} /$ liter. After $105 \mathrm{hr}$ of continuous operation at this dilution rate a microscopic examination of the culture revealed that a massive protozoan lysis had occurred. This cell lysis caused considerable amounts of debris to accumulate within the medium. This debris caused nonbacterial turbidity and clogged the cell counting instrument which made continued population censuing impossible with the techniques employed during periods when the debris was absent. Two other continuous runs of short duration (less than $200 \mathrm{hr}$ ) at low dilution rates resulted in a similar breakdown of the Tetrahymena population.

\section{DISCUSSION}

The interpretation of the batch and continuous culture data presented in the last section is facilitated by use of mathematical models. The synthesis of a model requires an explicit specification of the system mechanisms which integrates within a single formation all the important phenomena operative in that system. Figures 5, 6, and 12 show the dynamics of the population in a batch reactor. It is 


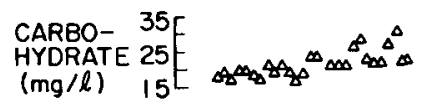

- Optical Density

- Biovolume

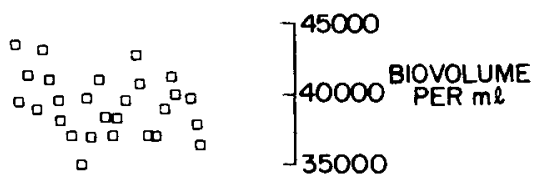

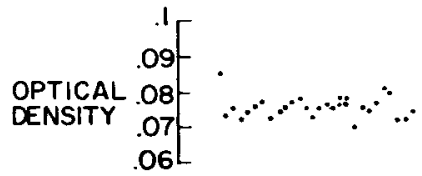
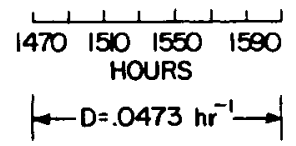

Fig. 11. Soluble carbohydrate, $O D$, and protozoan biovolume response during continuous culture at indicated dilution rate.

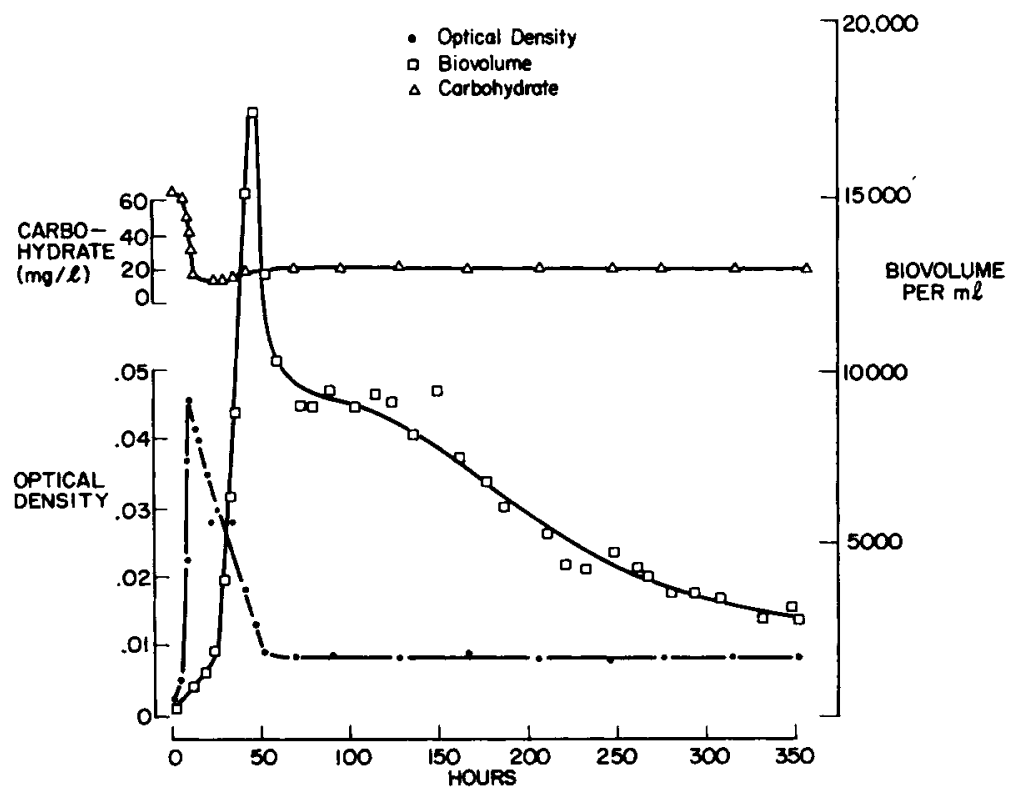

Fig. 12. Population and nutrient transients during batch culture. 
obvious that the decline of protozoan following the depletion of the bacterium is an important feature of the system which is not accounted for in eq. (5). The addition of a first order death term to eq. (5) permits consideration of this effect, and is included below in eq. (6) :

$$
\frac{d P}{d t}=\hat{\eta} \frac{H}{K_{H}+H} P-\frac{Q}{V} P-D_{P} P
$$

where $D_{P}$ is a death rate coefficient with units time ${ }^{-1}$.

Several batch runs have shown that the sucrose-Cerophyl substrate has a refractory fraction which is resistant to bacterial action. Furthermore, during the course of a batch run the refractory fraction increases following a minimum value. This phenomenon is presumably the result of excretions during the bacterial and protozoan metabolism; or the result of the break-up of bacterial and protozoan cells following growth. A separate material balance for the usable and refractory substrate and the bacterial prey is given below as eqs. (7), (8), and (9). The feedback features of the model are illustrated by Figure 13 .

$$
\begin{gathered}
\frac{d S_{u}}{d t}=\frac{Q}{V}\left(S_{u, i}-S_{u}\right)-\frac{\widehat{\mu}}{Y} \frac{S_{u}}{K_{S}+S_{u}} H+\frac{D_{H} H}{Y_{u}}+\frac{D_{P} P}{W_{u}} \\
\frac{d S_{r}}{d t}=\frac{Q}{V}\left(S_{r, i}-S_{r}\right)+\frac{D_{H} H}{Y_{r}}+\frac{D_{P} P}{W_{r}} \\
\frac{d H}{d t}=\hat{\mu} \frac{S_{u}}{K_{S}+S_{u}} H-\frac{\hat{\eta}}{W} \frac{H}{K_{H}+H} P-\frac{Q}{V} H-D_{H} H
\end{gathered}
$$

where

$S_{u}$ is the concentration of available substrate in the reactor $S_{u, i}$ is the concentration of available substrate in the feed $D_{H}$ is the first order death rate coefficient of the prey $Y_{u}$ is the amount of prey required per unit increase in $S_{u}$ $W_{u}$ is the amount of predator required per unit increase in $S_{u}$ $S_{r}$ is the concentration of refractory substrate in the reactor $S_{r, i}$ is the concentration of refractory substrate in the feed $Y_{r}$ is the amount of prey required per unit increase in $S_{r}$ $W_{r}$ is the amount of predator required per unit increase in $S_{r}$

Although the proposed prey-predator model given above as eqs. (6), $(7),(8)$, and (9) is nonlinear and therefore not readily integrated using analytical techniques, approximate solutions are possible using 


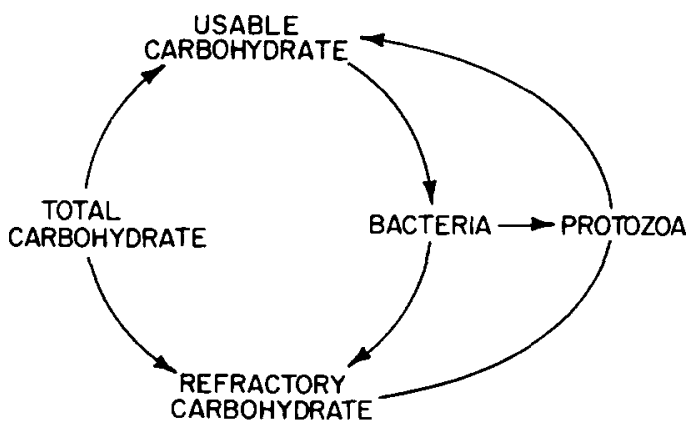

Fig. 13. Predator-prey interaction model with nutrient recycle feature.

numerical methods. It has been convenient to use the program CSMP (Continuous Systems Modeling Program) which is available on IBM computers for obtaining numerical solutions of prey-predator models. Curds ${ }^{15,16}$ has explained the programming procedures in detail for both three and six component population models.

Figure 14 shows the behavior of the numerically integrated model. These results are compared with batch population data presented in Figure 12. The stoichiometric and kinetic constants used during the simulation are listed in Table III. The initial conditions at the start of the integration were the same as the empirical initial conditions. The coefficients were selected to permit an optimum fit of

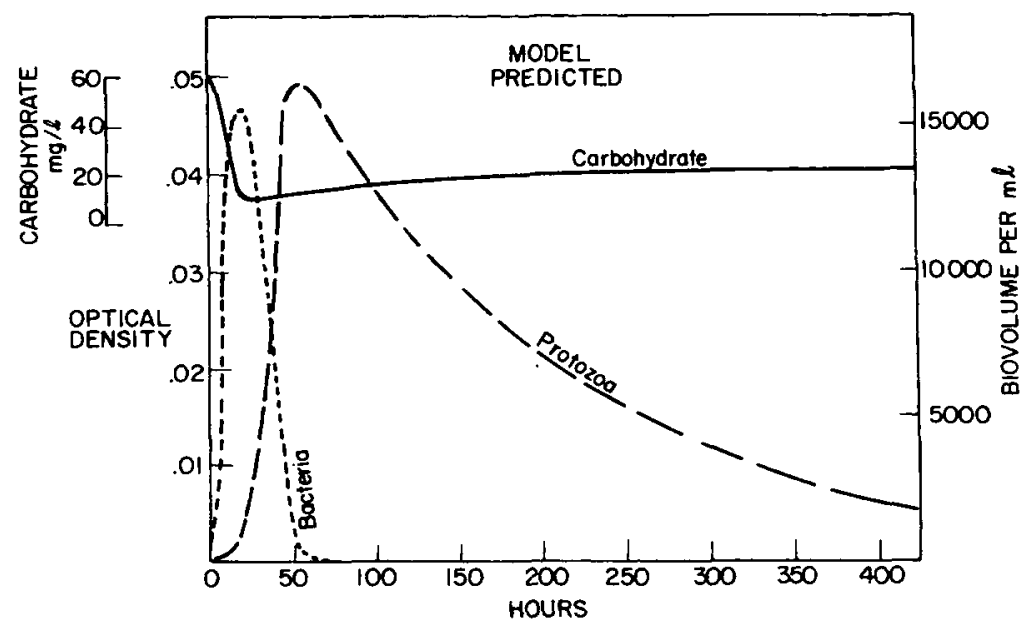

Fig. 14. Model predicted population and nutrient transients during batch culture. 
TABLE III

Kinetic and Stoichiometric Coefficients Used During Computer Simulations

\begin{tabular}{lc}
\hline Growth Rates & Stoichiometric Coefficients \\
$\dot{\mu}=0.56 \mathrm{hr}^{-1}$ & $Y=.00096 \mathrm{OD} / \mathrm{mg} / \mathrm{liter}$ \\
$\dot{\eta}=0.15 \mathrm{hr}^{-1}$ & $Y_{u}=0.005 \mathrm{OD} / \mathrm{mg} / \mathrm{liter}$ \\
Saturation Constants & $Y_{r}=0.0167 \mathrm{OD} / \mathrm{mG} / \mathrm{liter}$ \\
$K_{\mathrm{S}}=16 \mathrm{mg} / \mathrm{liter}$ & $W=450,000 \mathrm{biovolume} / \mathrm{OD}$ \\
$K_{H}=6.1 \mathrm{mg} / \mathrm{liter}$ & $W_{u}=5000 \mathrm{biovolume} / \mathrm{mg} / \mathrm{liter}$ \\
Death Rates & $W_{r}=2320 \mathrm{biovolume} / \mathrm{mg} / \mathrm{liter}$ \\
$D_{H}=0.0072 \mathrm{hr}^{-1}$ & \\
$D_{P}=0.0058 \mathrm{hr}^{-1}$ & \\
\hline
\end{tabular}

all the data in Figure 12 with the constraints being a reasonable agreement with the average values calculated from all batch experiments as summarized in Figures 1 through 4 . Of particular significance during the fitting procedure were characteristics of the dynamic behavior such as the time and amplitude of the peaks and the shape of the carbohydrate buildup and the protozoan and bacterial decline curves. In Table III the adoption of a maximum specific growth rate for the protozoan of $0.15 \mathrm{hr}^{-1}$ results in a calculated maximum growth rate of only $0.10 \mathrm{hr}^{-1}$ which is consistent with the experimental observations presented herein. This value of $0.15 \mathrm{hr}^{-1}$ is less than the value $0.2 \mathrm{hr}^{-1}$ reported by Curds and Cockburn ${ }^{9}$ for axenically grown and bacterially fed Tetrahymena. Figure 15 shows

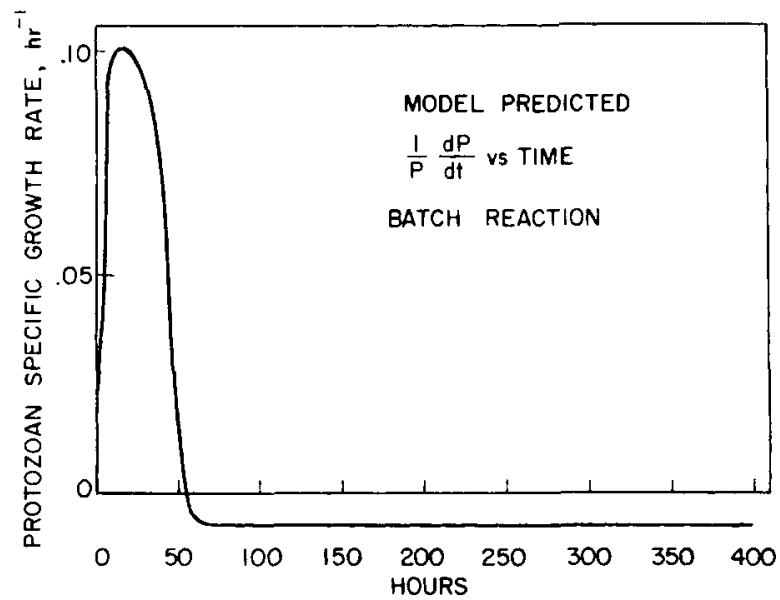

Fig. 15. Model predicted protozoan specific growth rate transients during batch culture. 
the instantaneous values of the protozoan specific growth rate during the batch culture.

Although the general agreement among the observed data and the simulated curves is satisfactory, the sharp peak and subsequent rapid decline of the protozoan biovolume was not possible to duplicate with the model. The data in Figure 6 suggest two distinct mechanisms for the protozoan biovolume decline. First, the relatively large cells in the absence of adequate food supply continue to divide and increase their swimming rate in seach of food. Qualitative microscopic observations of the Tetrahymena throughout their growth cycle in batch culture revealed that the cells swim much faster when food supplies are sparce. As the cells become smaller the rate of biovolume decline becomes less. The final phase of the decline is at the expense of actual cell numbers. The proposed model does not provide for changes in individual Tetrahymena cells, and therefore cannot precisely simulate the two-phase die away of the population. Improvements in the model formulation may result if the two component cell growth model as proposed by Williams ${ }^{17}$ were adopted.

It is observed that the maximum yield of the protozoan cells $(0.73$ $\mathrm{g}$ of protozoa/g of bacteria) is maintained for only a few hours. High yields of Colpoda ( $0.78 \mathrm{~g}$ of protozoa/g of bacteria) were also reported by Proper and Garver ${ }^{8}$ when the cells were grown in batch culture. The yield of Tetrahymena in a two-stage continuous culture was measured by Curds and Cockburn. ${ }^{10}$ In this case relatively small cells were maintained at steady state resulting in a yield of only $0.54 \mathrm{~g}$ of protozoa/g of bacteria.

The kinetic and stoichiometric coefficients in Table III were used during computer simulations of each continuous run by adopting the appropriate dilution rate for each case.

Figure 16 shows the computed results for the $D=0.136 \mathrm{hr}^{-1}$ run and should be compared with the data observed under a similar dilution rate shown in Figure 7. The computed protozoan population approached a steady biovolume density of about 13,000 per ml. At all instances during the simulation the rate of change of protozoan was negative. The observed Tetrahymena population showed positive as well as negative rates of change during a decline to less than 10,000 biovolume units $/ \mathrm{ml}$. The observed peak at 120,000 biovolume units $/ \mathrm{ml}$ was not duplicated by the model. The computer predicted profiles of bacterial optical density and total carbohydrate agree reasonably well with the empirical data. It is apparent that the Tetrahymena can multiply at a specific growth rate much greater 


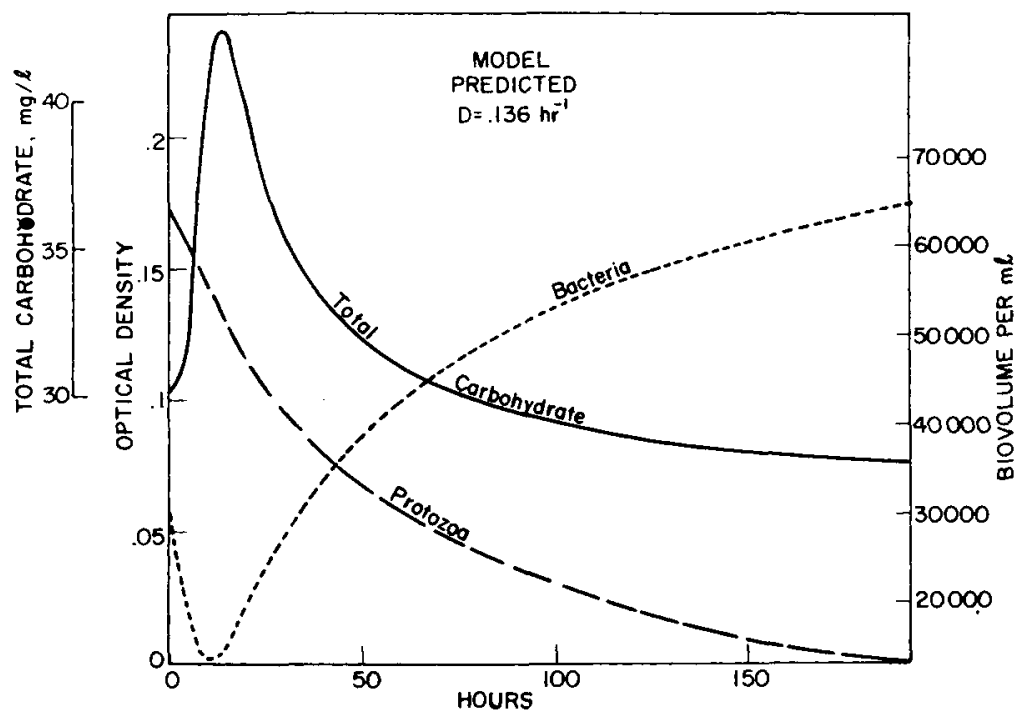

Fig. 16. Model predicted soluble carbohydrate, OD, and protozoan biovolume responsed during continuous culture at indicated dilution rate.

than $0.136 \mathrm{hr}^{-1}$. However increasing the value of $\hat{\eta}$ in the model will result in high stationary protozoan levels within a relatively short time period.

Figures 17 and 18 show the results of a computer simulation of the continuous run having a dilution rate of $0.0715 \mathrm{hr}^{-1}$. The model coefficients employed are the same as those used in other simulations and are listed in Table III. The computed results for this case resemble the data shown in Figure 9 for the first few hours of the run. While the computed results continue oscillating indefinitely, the observed fluctuations dampen after approximately 200 hr of culture. During low bacterial densities, the computed results predict very rapid increases of total carbohydrate. For both this and the previous case, observed optical density minimums were always greater than the predicted values which resulted in low carbohydrate levels within the reactor. The model can be made to exhibit damped oscillatory behavior by decreasing the value of $\hat{\eta}$ while keeping other parameters fixed. However this modification of $\hat{\eta}$ further displaces the computed results from the observations during the $D=0.172$ hr-1 run.

Although formalized search techniques were not employed, it seems that it is not possible to adjust the model coefficients in a manner which will allow satisfactory simulation of data observed 


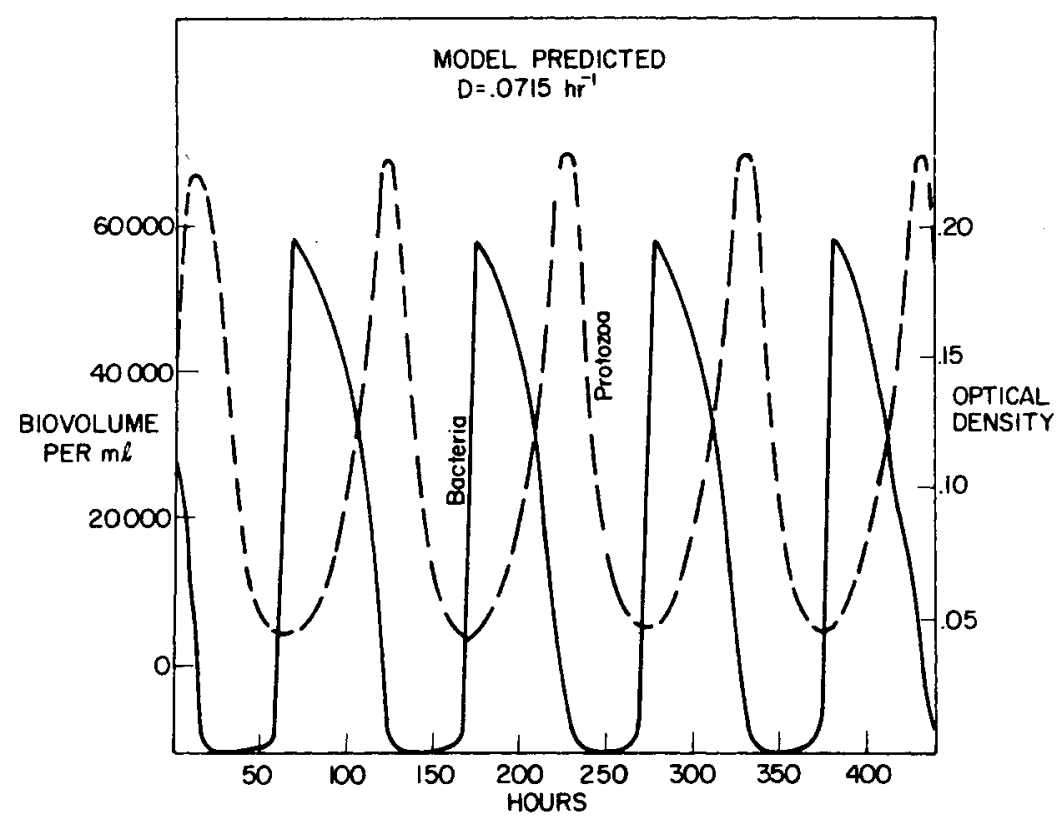

Fig. 17. Model predicted OD and protozoan biovolume response during continuous culture at indicated dilution rate.

during all dilution rates employed during this study. The incorporation of time delay features in the growth expressions for the bacterium and the protozoan may improve the predictive capabilities of the model. Bungay and Paynter ${ }^{18}$ have studied prey-predator models with first order delays between substrate changes and microorganism growth responses. They have shown that high substrate levels during prey troughs are reduced considerably by addition of the time delay feature. The prolonged low-level of protozoan following the initiation of the $D=0.0825 \mathrm{hr}^{-1}$ run further supports the notion that time-delayed growth responses are important in the cultures.

The breakdown and lysis of the Tetrahymena at low optical density levels was observed during three continuous runs. Since the known nutritional requirements of the protozoan were available within the culture medium at all times, no explanation of the phenomenon can be given at this time. However, it is suspected that an internal control mechanism may be operative which causes a reduction of the Tetrahymena population during periods of extreme food shortages. The debris which accumulated following protozoan lysis had an appearance similar to that of activated sludge floc. It was observed 


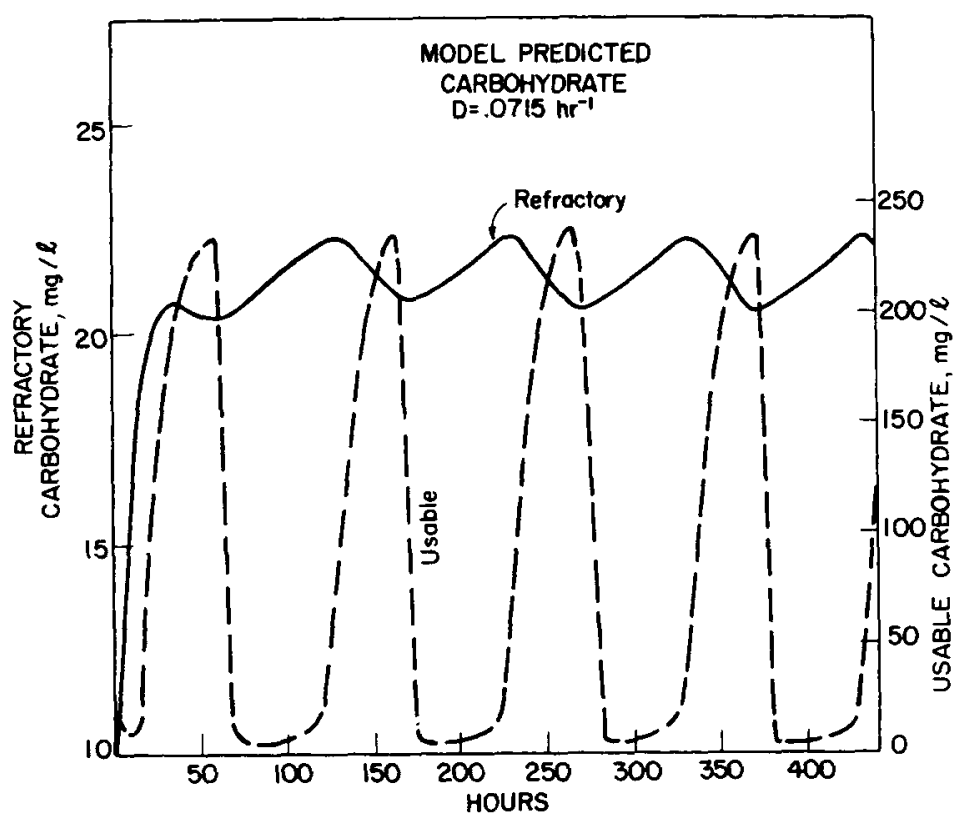

Fig. 18. Model predicted usable and refractory soluble carbohydrate response during continuous culture at indicated dilution rate.

that bacterium and the remaining protozoan tended to attach to particles of debris where food levels were higher. Streak plates of the culture during this period did not reveal the presence of any contaminating organisms.

\section{CONCLUSIONS}

The mathematical model studied herein (eqs. (6), (7), (8) and (9)) is capable of simulating the general behavior of the batch growth of Aerobacter aerogenes and Tetrahymena pyriformis subject to the experimental conditions imposed. The model is not able to duplicate the two-phase decline of the protozoan in the absence of bacteria.

The model examined is not able to account for the transient response of the population during continuous culture at all the different dilution rates. It is proposed that higher order effects such as time delays of growth rate changes in response to environmental changes and self-regulating and adaptive ability of the Tetrahymena are important phenomena which cannot be disregarded.

The work reported in this paper was supported by the University of Michigan Sea Grant Project. 


\section{References}

1. A. J. Lotka, Elements of Physical Biology, The Williams and Wilkins Co., Baltimore, Md., 1925.

2. V. Volterra, Mem. Acad. Lineii Roma, 2, 31 (1926).

3. J. F. Drake, J. L. Jost, A. G. Fredrickson, and H. M. Tuschiya, The Food Chain, Bioregenerative Systems, A conference held in Washington, D.C., November 15-16, 1966 sponsored by NASA and the American Institute of Biological Sciences, NASA SP-165), Library of Congress No. 68-60345, U.S. Government Printing Office, Washington, D.C., 1966.

4. R. P. Canale, Biotechnol. Bioeng., 11, 887 (1969).

5. H. R. Bungay III and D. M. Cline, "Revised models for microbial interactions," paper presented at American Chemical Society Meeting, Chicago, Sept., 1969.

6. J. N. Yang and C. R. Weston, "A mathematical model of the effect of a Predator on species diversity," Tech. Rep. 32-1359, Jet Propulsion Laboratory, Calif. Inst. of Technol., Pasadena, Calif., 1969.

7. D. Herbert, R. Elsworth, and R. C. Telling, J. Gen. Microbiol, 14, 601 (1956).

8. G. Proper and J. C. Garver, Biotechnol. Bioeng., 8, 287 (1966).

9. C. R. Curds and A. Cockburn, J. Gen. Microbiol., 54, 343 (1968).

10. C. R. Curds and A. Cockburn, J. Gen. Microbiol., 66, 95 (1971).

11. R. P. Canale, Biotechnol. Bioeng., 12, 353 (1970).

12. H. S. Ducoff, S. M. Williams, T. B. Syn, and B. D. Butler, J. Protozool, $11(3), 309$ (1964).

13. T. D. Lustig, "Predator-prey interactions in an experimental protozoan community (Tetrahymena pyriformis-Aerobacter aerogenes)," Tech. Rep. of Univ. of Mich. Sea Grant Program, Ann Arbor, Mich., 1971.

14. A. F. Gaudy, Ind. Water Wastes, 7, (1), 17 (1962).

15. C. R. Curds, Water Res., 5, 793 (1971).

16. C. R. Curds, Water Res., 5, 1049 (1971).

17. F. M. Williams, in, Systems Analysis and Simulation Ecology, B. C. Patten, Eds., Academic Press, New York, 1971.

18. H. R. Bungay III and M. J. B. Paynter, "Models for multicomponent microbial ecosystems," paper presented at the 69th National Meeting of the American Institute of Chemical Engineers, Cincinnati, Ohio, 1971.

Accepted for Publication February 5, 1973. 